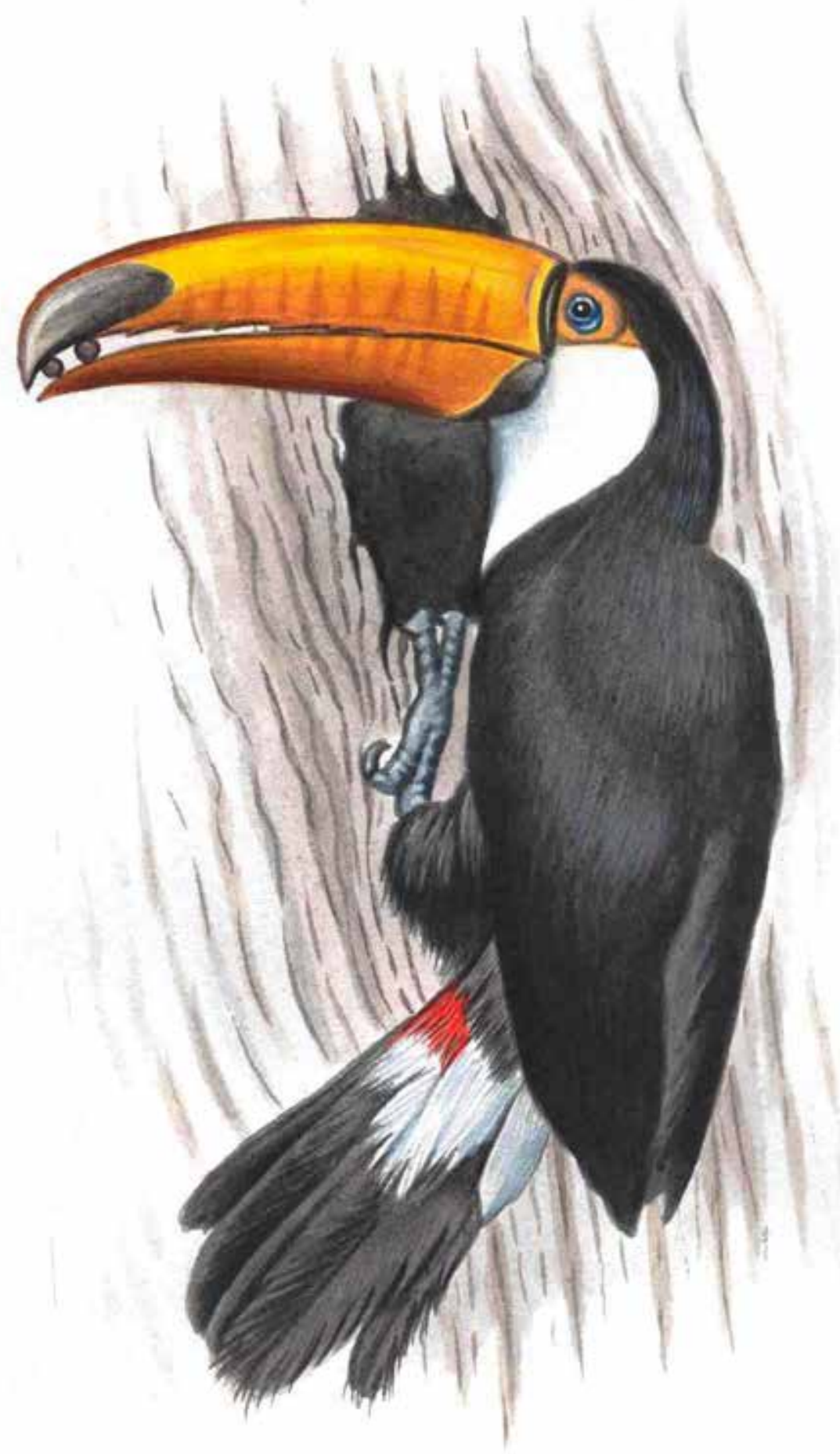

Leonardo González Galli

Profesor Investigador Univesidad de Buenos Aires

Técnica: Acrílico sobre Cartón 


\section{DE LA DIVERGENCIA A LA COMPLEMENTARIEDAD: UNA APROXIMACIÓN HISTÓRICA A LA ECOLOGÍA}

Fecha de recepción: 24 de marzo de 2014 Fecha de aprobación: 03 de junio de 2014

\section{Jairo Robles-Piñeros ${ }^{1}$}

\section{Resumen}

Desde sus inicios, la construcción del corpus conceptual y la definición del campo de acción e investigación de la ecología han sido temas de bastante discusión en los ámbitos histórico e intelectual; a pesar de poseer un espacio definido dentro de los límites de la investigación, es un hecho que estas supuestas fronteras no se han hecho tan notorias durante su conformación como disciplina científica a través de la historia, así como tampoco en la actualidad del mundo académico. No obstante, se propone hacer una aproximación a la construcción conceptual de la ecología con el fin de reflexionar y entender los diferentes factores históricos e intelectuales a los que se vio enfrentada en su camino para la consolidación científica. También, se analizan los alcances de otras disciplinas científicas en la construcción conceptual de la ecología y en especial como un enfoque producto de la convergencia con otras áreas del conocimiento; de la misma manera, se aborda la cuestión del estatus de la ecología dentro de las ciencias, así como la necesidad de un espacio de encuentro entre diferentes enfoques, en la búsqueda de la construcción de un puente de complementariedad entre las ciencias y las humanidades.

\section{Palabras clave}

Conciencia ecológica, consiliencia, historia de las ciencias, pensamiento holístico,

\section{Abstract}

The building of a conceptual corpus and the definition of the action field and investigation of the ecology, since its beginning have been a topic of very hard discussion in the historical and intellectual field; despite of have a defined space into the edges of investigation, in deed these frontiers aren't so notorious in the conformation as scientific discipline through the history, neither in the actuality of the academically world. However, it's proposed make a historical approach to the conceptual building of the ecology with the objective of understand historical and intellectual factors which was faced in its way to the scientific consolidation. Moreover, scopes the historical approaches of another scientific disciplines in the conceptual building of ecology and as a approach product of the convergence with another areas of knowledge; in that way to open the discussion about the status of the ecology as a science, as well as the need of one meet space between different approaches not only scientific but also socials in the searching of the construction of a complementary bridge between the sciences and humanities.

\section{Keywords}

Consilience, ecologic conscience, history of science, holistic thinking.

1 Licenciado en Biología. U. P. N. Docente Colegio San Viator. Correos electrónicos: jairohxcbogota@gmail.com, jairorude25@hotmail.com. 


\author{
"La mayor empresa de la \\ mente siempre ha sido \\ y siempre será el intento de \\ conectar las ciencias con las \\ humanidades."
}

\section{Edward O. Wilson}

¿Por qué a pesar de estar en pleno siglo XXI se observa un límite entre algunas ramas de la ciencia y, por supuesto, las humanidades? ¿Es acaso una tendencia de la humanidad abrir fronteras entre una y otra cosa con el fin de comprender mejor su naturaleza, y así estar en la capacidad de explicarlas? 0 , ¿es cuestión de entender cómo se han construido los diferentes saberes a través de la historia y los obstáculos a los que estas disciplinas tuvieron que enfrentarse, lo que pudiera dar una pista para responder a esta situación?

Cada sociedad, a través de la historia, ha hecho una representación propia de su relación con la naturaleza; el interés por un acercamiento a la explicación de los fenómenos naturales y el lugar que el hombre ocupa en la naturaleza ha sido, desde tiempos inmemoriales, una premisa en la construcción del conocimiento en las colectividades. Para muchos pueblos antiguos, las representaciones de la naturaleza se conformaron a partir de la concepción de prioridad en la recolección de datos como necesidad para la supervivencia de la especie; es decir, desde el precepto de que si no se conoce aquello que les rodea no sería posible sobrevivir a los cambios que el ambiente impone.

Entonces, se observa una cercana relación entre las pautas sociales y humanas dentro de los avances científicos y tecnológicos en las diferentes etapas de la sociedad, el límite entre lo que le compete a las ciencias y a las humanidades no se percibe en las sociedades antiguas. Cuando Snow (1959) en su discurso declara "la separación entre ciencias y letras, o ciencia y humanidades"; hace hincapié en la disyunción entre estos dos enfoques. Pero, ¿es esto cierto? ¿No existen acaso cientos de aproximaciones científicas por parte de personajes no "científicos"? Nada tiene de extraño entonces escuchar a conocidos artistas remitiéndose a la ciencia como marco intelectual e, incluso, como fuente de inspiración.

La ecología ha tenido un papel primordial en la transformación de cómo la humanidad observa y comprende su entorno natural inmediato; como rama de la biología, esta se ha visto constantemente vulnerada por todos aquellos cambios en el pensamiento derivados del contexto del cual forma parte; es decir, a través de su historia y su consolidación ha demostrado ser un enfoque en el que convergen no solo disciplinas científicas, sino que además y en pleno comienzo de un siglo, se hace cada vez más evidente el traspaso de los límites con las humanidades, como necesidad para la búsqueda de soluciones a los problemas surgidos en siglos pasados.

Basta con echar un vistazo al pasado para tener un panorama general del proceso de construcción y consolidación de la ecología como disciplina científica, y entender el porqué de su naturaleza interdisciplinar al encontrar sus orígenes en aproximaciones que provenían de diferentes enfoques diametralmente opuestos en apariencia, pero convergentes en el sentido general. Desde la antigua Grecia, pasando por la revolución industrial y científica, hasta la actualidad la ecología se ha caracterizado por poseer un enfoque lleno de contribuciones; por esto, se hace necesario realizar un acercamiento histórico para comprender su naturaleza como corriente de conocimiento y práctica científica.

\section{Aristóteles y el pensamiento griego}

Heredero de la tradición de la historia natural y de la búsqueda de la posición de la humanidad dentro del mundo natural, el trabajo de Aristóteles y sus contemporáneos (como Plinio el Viejo) se caracterizó por enfocarse en explicaciones teocéntricas y creacionistas de la naturaleza; esta y cada uno de los organismos que la conformaban evidenciaban una creación lineal y jerarquizada sobre la cual se encontraba el hombre como el organismo "superior"; aun así, esto se tradujo en afán por conocer este entorno inmediato y llevó a la descripción y clasificación de las especies en una forma que permitiera su entendimiento, de allí derivó la noción de continuidad dentro del mundo natural. El trabajo, llevado a cabo durante esta época no solo por Aristóteles sino por otros, como es el caso de Teofrasto con su De historia plantarum, conlleva a identificar las primeras nociones de equilibrio dentro de los organismos, además de su relación entre sí y con el hombre (Deléage, 1993).

\section{La concepción de naturaleza de Linneo}

No es de extrañar que durante un lapso considerable el avance en cuanto a las observaciones de la naturaleza y la postulación de enfoques alternos a las aproximaciones hechas por los gigantes clásicos se vieran diezmados a causa de la presión eclesiástica ejercida sobre la libertad de pensamiento en todo el mundo occidental. Además de los trabajos de Aldrovandi y Gesner, el avance en la descripción de la naturaleza no tuvo mayor impulso sino hasta finalizado el siglo XVII (Gould, 2004). En el trabajo desarrollado para el contexto del siglo XVIII y la publicación de Systema Naturae (imagen 1), se evidencian relaciones recíprocas en la organización de la naturaleza a 
pesar del uso de un sistema jerárquico de origen inmutable, puesto que al proponer el método de clasificación, se hace referencia a las similitudes entre las especificidades de cada uno de los organismos.

Se propone una economía de la naturaleza, que según Linneo se visualiza en la disposición que los seres naturales exhiben, atendiendo a fines comunes y relaciones interdependientes; respondiendo así, a la innata capacidad de propagación de las especies y su distribución geográfica, sin atentar contra el equilibrio y los recursos de los cuales dependen. El concepto linneano propone la noción de balance, al mencionar que la naturaleza tiene el papel de policía, cuyo objetivo es velar por el mantenimiento del equilibrio natural (Deléage, 1993).

Un dato interesante de este trabajo es que Linneo incluye al hombre dentro de las categorías Quadripedia (animales cuadrúpedos) y Anthropomorpha junto con otros primates (orden que Linneo crea para asignar al humano y otros simios) (imagen 2), esto sin duda fue un avance en el pensamiento, ya que se reconoce como el primer paso en el camino hacia el rompimiento de la tradición antropocéntrica de clasificación natural; reconociendo al ser humano como un animal y dándole así su nombre científico Homo sapiens.

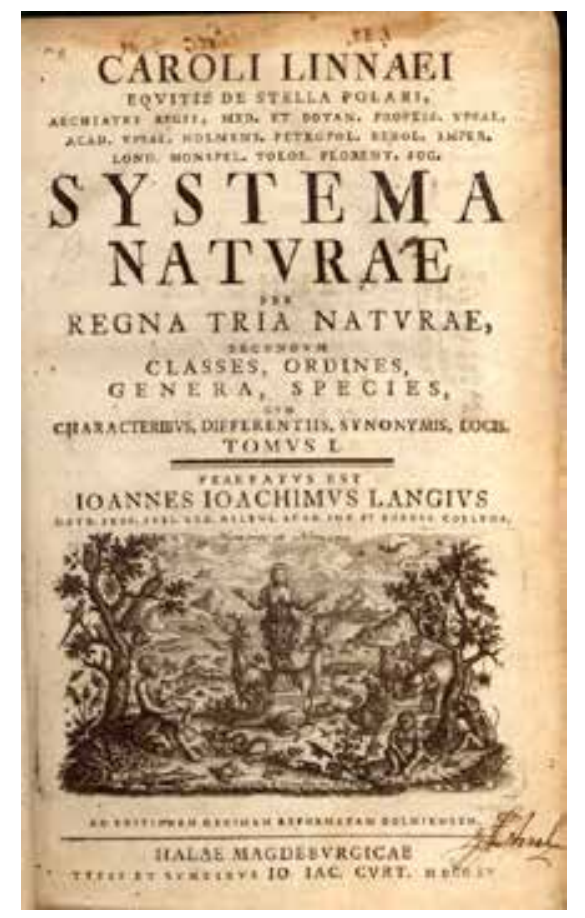

Imagen 1. Frontispicio de Systema Naturae de Carl Von Linne.

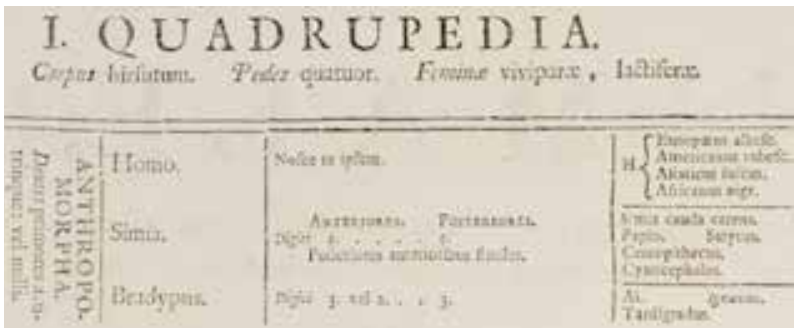

Imagen 2. Dentro de la categoría Quadrupedia (Cuadrúpedos), Lineo incluye al hombre.

\section{Humboldt, las plantas y el pensamiento poblacional de Darwin y Wallace}

Al dar comienzo a la explosión colonizadora y la creciente producción de productos, se exigió un incremento en la demanda y uso de los recursos naturales, la visión que se tenía sobre el mundo natural tomó un nuevo giro; el interés por conocer y comprender aspectos importantes acerca de la naturaleza se incrementó. De igual manera, la revolución de pensamiento y la instauración del método científico propusieron una nueva forma de comprensión del mundo (Barra, 1996); con el trabajo realizado por Humboldt y sus allegados durante la segunda mitad del siglo XIX, en las expediciones botánicas en el nuevo mundo, se abrió una nueva esfera de aplicación de la investigación científica dentro del conocimiento biológico.

Por otra parte, Lamarck (1986) propuso que, dado que el ambiente se halla en constante transformación, los organismos necesitan cambiar y realizar un esfuerzo para mantenerse y sobrevivir; es decir, plantea un mecanismo de evolución y una de las principales bases de la ecología puesto que involucra las relaciones de los organismos y su entorno. Al darle una nueva organización al marco de estudio de los organismos como especies, los trabajos de Darwin y Wallace, responden a la necesidad de integración de los saberes obtenidos durante siglos respecto a las especies y su papel dentro del ambiente; además, se explicitó la influencia que las variaciones ambientales tienen sobre los organismos y las modificaciones en las poblaciones y su distribución. Cuando se plantea que existen variaciones a nivel individual y la selección natural tiene lugar en las poblaciones, se da un rompimiento con la tendencia creacionista y estática que durante varios siglos había predominado. Pero además, al hacer mención de la existencia de factores ambientales influyentes en las dinámicas y comportamientos de las especies, se abre un nuevo camino para la investigación del mundo natural y el nacimiento de una nueva rama del conocimiento (Deléage, 1993). 


\section{Haeckel y la invención de la Ökologie}

Dentro de las sociedades europeas en las que se dio un desarrollo industrial y una dinámica económica expansionista veloz, se evidenció la necesidad de una comprensión más profunda del funcionamiento de la naturaleza, con el objetivo explícito de aumentar la eficacia de la explotación productiva. De esta necesidad nacerá la ecología, con la idea de que el mismo equilibrio que rige la naturaleza también rige a las sociedades, y así se darán las bases para esta disciplina. Hacia finales del siglo XIX, Ernst Haeckel, gran defensor de las ideas darwinianas, acuñó el término ecología definiéndola en palabras de Deleage (1993, p. 73) como: "La ciencia de las relaciones de los organismos con el mundo exterior, en el que podemos reconocer de una forma amplia la lucha por la existencia".

Se produce así, el surgimiento de una nueva forma de ver el mundo y sus dinámicas, no solo la biología y en su defecto la fisiología podría dar las explicaciones del mundo natural; el enfoque ecológico tiene en cuenta necesariamente todas las relaciones encontradas entre los organismos y su entorno; por ello, la intervención de otras disciplinas científicas comienza a desempeñar un papel importante en la construcción del conocimiento en biología.

\section{Möbius, los ecosistemas y la humanidad dentro de la naturaleza}

Desde los aportes hechos por la química, la física y la termodinámica, la concepción de continuidad dentro de la naturaleza tomó un giro revolucionario, sobre cómo se comprenden sus dinámicas. Möbius, en su trabajo Die Auster und die Austernwirtschaft en el que expuso las características ambientales para la cosecha de ostras, y la distribución geográfica de algunas plantas, propuso la noción de continuidad y ciclos sin fin dentro de cada espacio natural, al comprender que dentro de la naturaleza cada proceso químico y físico llevado a cabo es, en esencia, un bucle que vuelve al inicio, un constante retorno al punto de partida.

Esta visión de ciclos y continuidad fue fundamental en la estructuración del concepto de ecosistema; ya que al plantearse de esta forma el abordaje del mundo natural y teniendo en cuenta la noción de una humanidad perteneciente a la naturaleza, se establece un nuevo grado de organización de la misma, en el que el hombre adquiere un papel importante dentro de cada una de sus dinámicas, visto desde cómo lleva a cabo la producción y en su relación con el resto de organismos.

\section{Odum y la ecología como una ciencia consciente de sí misma}

Al tomar seriamente los aportes de la termodinámica en la construcción de conocimiento de la ecología, se adquiere un nuevo patrón de estudio, cuando se introduce la noción de flujo de energía en los sistemas vivos y el entorno del cual forman parte; el estudio sobre las relaciones tróficas se convierte entonces en el principal enfoque práctico de la ecología para el siglo XX, este será el foco de trabajo de biólogos y ecólogos, en aras constatar el equilibrio mantenido dentro de cada sistema mediante el proceso de transformación de la energía.

Mediante estas intervenciones, la concepción de equilibrio toma un nuevo significado al observar el impacto que la humanidad como especie ha tenido durante su explosión demográfica luego del siglo XVIII (Crosby, 1999), la ecología se convierte así en una ciencia consciente de sí misma, ya que nace de la necesidad de dar una explicación desde la comprensión humana a las dinámicas naturales, teniendo en cuenta que este conocimiento está forjado desde la experiencia de la humanidad como especie que forma parte de un ambiente inmediato y se rige a sus dinámicas, y además lo comparte con miles de otras formas de vida.

La concepción unitaria del entorno y el esteticismo de las especies a través del tiempo fueron premisas de la Antigüedad, pero en el siglo XXI aún se encuentran grandes rasgos de este pensamiento dentro de la enseñanza de las ciencias en la escuela (Hernández, 1987); se plantea desde los nuevos caminos de la ciencia y la nueva filosofía integradora científica, que el conocimiento científico es inseparablemente, experiencia de la realidad e intuición creadora, hay una búsqueda de síntesis integradora entre la razón y la sensibilidad.

Se debe reconocer, entonces, que la ecología como todas las disciplinas científicas y como una empresa de construcción humana, se encuentra siempre en constante reevaluación y, por supuesto, reestructuración, tanto conceptual como metodológica. En la discusión sobre el estatus de la ecología como ciencia, Deleage (1993) plantea:

\section{Obstáculos de la ecología para conformarse como ciencia:}

1. La exigencia enciclopédica. Toda la reflexión se encuentra deformada por el sesgo de la lengua y el etnocentrismo que introduce esta situación. 
2. Un obstáculo ligado a la heterogeneidad de una ciencia en plena evolución teórica y confrontada a una diversidad de registros y de terrenos muy grande. ¿Sobre qué corpus trabajar? Y, ¿cuál es el límite?

3. Las implicaciones sociales más importantes de la ecología. La ecología se encuentra llena de interrogantes metacientíficos.

La ciencia tiene que evolucionar entre dos necesidades, contradictorias en apariencia: la de la especialización para profundizar los conocimientos específicos o regionales de la realidad, y la de la globalización indispensable para la comprensión del funcionamiento del conjunto. En el mundo científico se siente la necesidad de un saber unificado que articule dentro de una visión global, los diversos enfoques del mundo. En las sociedades, llevadas por la dinámica conquistadora y depredadora del capitalismo, se expresa la necesidad de una comprensión más profunda del funcionamiento de la naturaleza, con el objetivo explícito de extender y aumentar la eficacia de su explotación. La ecología nacerá de esta necesidad. La idea de que el mismo equilibrio natural rige la marcha de la sociedad y la de la naturaleza sentará las bases de la ecología, como lo hizo en el caso de la economía. (Deléage, 1993, p. 66-67).

\section{El paradigma ecológico}

Es evidente que conocer solo las propiedades de cada parte que conforma un sistema como una entidad separada no dará jamás una explicación completa del nivel objeto de estudio, porque al analizar el nivel superior desde un enfoque diferente, estas partes se combinan e interactúan (Wilson, 1999). Entonces, han de incluirse también dichas interacciones como aspectos esenciales de una explicación adecuada del nivel emergente. No es posible, entonces, destacar una explicación desde un enfoque que no tenga en cuenta el sistema en general y todas las posibles interacciones que se puedan evidenciar. Porque si dichas propiedades emergentes simplemente no existen en un nivel de organización menor, y tampoco pueden ser inferidas, no es posible que haya cabida a un razonamiento basado en la reducción para la comprensión (Bowler y Rhys, 2007).

Cuando se menciona un paradigma desde el punto de vista de las ciencias, se hace referencia al conjunto de teorías que dan como resultado el objeto de estudio; la ecología como disciplina de la biología se encuentra en proceso de configurar su propio paradigma. Esto visto desde la implicación filosófica que le atañe a la humanidad desde el entendimiento y la comprensión de la relación mantenida con la naturaleza (Barra, 1996).
Al plantear un paradigma ecológico se propone una transformación del pensamiento y cambio epistemológico y un nuevo abordaje de las ciencias, que ayuda a clarificar el significado del movimiento sistémico en el seno de la ciencia actual.

La ecología es una revolución epistemológica, es decir, es otro modo de pensar. Es otra lógica, no es la lógica causal clásica. La idea de que todo incide sobre todo nos lleva a reinventar la totalidad de las cosas en cada momento. Esto es otro tipo de lógica, que se inscribe en un paradigma distinto y para mí, la ecología básicamente es esto, un nuevo paradigma. (Paniker, citado por Hernández, 1987, p.50-51).

La alfabetización científico-biológica se hace necesaria al pretender una mejor comprensión y construcción conceptual de las ciencias y el desarrollo de actitudes que se traduzcan en comportamientos favorables hacia lo vivo, es decir la formación de seres conscientes de su papel dentro del planeta y de la responsabilidad de conservarlo. Sin embargo los bloqueos son múltiples, la estructura general del sistema científico, la división y compartimentación del saber académico, la jerarquización de los contenidos científicos y su desligamiento con el contexto social y cultural, hacen que se vea diezmado el abordaje holístico dentro el ámbito educativo. Desde hace ya décadas, en el ámbito de la enseñanza de las ciencias naturales, se evidencia un estado de crisis, que en la actualidad sigue sin solución, básicamente porque el tipo de enseñanza impartida en la escuela es de carácter anacrónico y descontextualizado de la realidad inmediata del estudiante (Robinson, 2009).

Es claro para todos que la fusión de saberes como en el Renacimiento es hoy una empresa imposible de lograr; el gran mundo de especialización es bastante voluminoso. Sin embargo, es necesario hacer que los diferentes saberes comuniquen, sin reducirse los unos a los otros; es decir que sin buscar un principio unificador de todos los conocimientos se aspire a un diálogo entre disciplinas sobre la base de un pensamiento complejo (Dennet, Deutsch y Diamond, 2007). Ni todo es biología, ni todo es ecología, ni mucho menos sociología; el paradigma ecológico debe ensalzar la necesidad de una permeabilidad entre ciencias, letras y artes como una exigencia de este siglo en el que, en palabras de Brockman (1996), "[...] está más allá de la revolución científica”, resaltando con esto que no solo desde la ciencia dura se puede ofrecer respuestas a las cuestiones centrales de la humanidad y por lo tanto, implica un nueva forma de abordarlas desde la convergencia de saberes, el reencuentro de disciplinas y el trabajo colaborativo de los enfoques humanistas y científicos. 
La unidad del conocimiento como una necesidad dada por la relación entre ciencias y humanidades, es aún una empresa con bastantes obstáculos de tipo social, cultural, político y filosófico; pero si en algo se debe avanzar como premisa para la constitución de este puente de complementariedad, (entendido como el uso de un lenguaje común entre dos enfoques explicativos de un mismo fenómeno) (Azkonobieta, 2005), es en la búsqueda de un espacio conjunto en el que se decanten los aportes de la ciencia y las humanidades. El paradigma ecológico no debe dar la espalda a ninguna de estas dos aproximaciones; por el contrario, debe tomar una posición en la que haga, como se dice popularmente, "sacar lo mejor de dos mundos" y, así, forjar los cimientos de tan esperado puente.

\section{Referencias bibliográficas}

Azkonobieta, T. (2005). Evolución, desarrolloy (auto) organización. Un estudio sobre los principios filosóficos de la evo-devo. Tesis doctoral no publicada. Universidad del País Vasco, Donostia, San Sebastián.

Barra, A. (1996). Antiecología. Apuntes de una filosofía y paradigma ecológico. Buenos Aires: Espacio Editorial.

Bowler, P. y Rhys, I. (2007). Panorama general de la ciencia moderna. Barcelona: Editorial Crítica.

Brockman, J. (1996). La tercera cultura. Más allá de la revolución científica. Barcelona: Tusquets Editores.

Crosby, A. (1999). Imperialismo ecológico. La expansión biológica de Europa 900-1900. Barcelona: Editorial Crítica "Libros de historia".

Deléage, J. (1993). Historia de la ecología. Una ciencia del hombre y de la naturaleza. Barcelona: Icaria Editorial S.A.

Dennett, D.; Deutsch, J. y Diamond, A. (2007). El nuevo humanismo. Y las fronteras de la ciencia. Barcelona: Editorial Kairós.

Gould, S. (2004). Érase una vez el zorro y el erizo. Las humanidades y las ciencias en el tercer milenio. Barcelona: Editorial Crítica.

Hernández, A. (1987). El paradigma ecológico: Marco para la investigación de la enseñanza-aprendizaje en ciencias. Revista Enseñanza de las Ciencias 5 (Extra 1), 50-51.

Lamarck, J, B. (1986). Filosofía zoológica. Editorial Alta Fulla, Barcelona.

Paniker, S. (1987). Ensayos retroprogresivos. Barcelona: Editorial Kairós S.A.
Robinson, K. (2009). The element. How finding your passion changes everything. Nueva York: Penguin Books.

Snow, C, P. (1959). The two cultures and the scientific revolution. The rede lectures, Cambridge University Press. New York.

Wilson, E. (1999). Consilience. La unidad del conocimiento. Barcelona: Galaxia Gutenberg, Círculo de Lectores. 